\title{
ESTIMATION OF RISK
}

\author{
Helen M Kingston
}

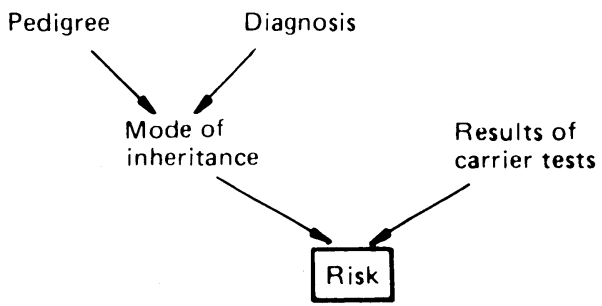

This chapter gives some examples of the risk of developing or transmitting a mendelian disorder. The mathematical risk calculated from data on a pedigree may often be modied by additional information from specific tests to detect carriers. Risk can be expressed as either a percentage or a fraction.

\section{Autosomal dominant disorders}

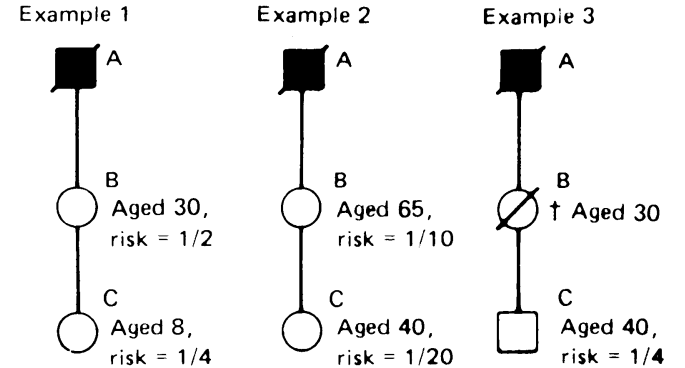

The $50 \%$ risk of developing a condition for the offspring of an affected person may be modified by age in disorders whose onset is in adult life, such as Huntington's chorea. In examples 1 and 2 the risk to person B of developing Huntington's chorea is still $50 \%$ at age 30 years, but by the age of 65 the residual risk to a healthy person has fallen to $10 \%$. The risk to person $\mathrm{C}$ therefore falls from $25 \%$ in example 1 to $5 \%$ in example 2 . In example 3 the risk for $\mathrm{C}$ cannot be reduced below $25 \%$ because parent $\mathrm{B}$, although clinically unaffected, died aged 30 while still at $50 \%$ risk.

Example 4

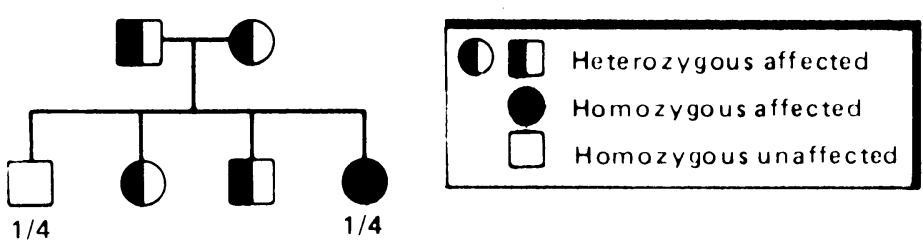

Example 5

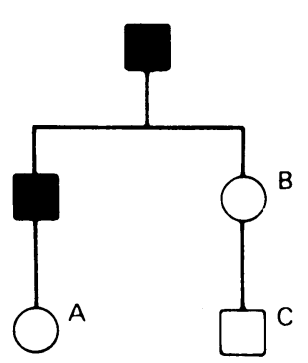

\begin{tabular}{ccc}
\hline Person & $\begin{array}{c}\text { Risk of having } \\
\text { inherited gene } \\
(\%)\end{array}$ & $\begin{array}{c}\text { Risk of developing } \\
\text { disorder } \\
(\%)\end{array}$ \\
\hline A & 50 & 40 \\
C & 8 & $6-7$
\end{tabular}

When both parents have the same autosomal dominant disorder, the risk to the offspring will be high (example 4). Only one in four children will be unaffected, and one in four will be homozygous for the mutant gene, which may cause severe disease, as in familial hypercholesterolaemia or achondroplasia.

Reduced penetrance also modifies simple autosomal risk. Example 5 shows the risks for a disorder with $80 \%$ penetrance in which only $80 \%$ of gene carriers develop the disorder. Although clinically unaffected, person B may still carry the mutant gene, and there is therefore a fairly small risk of her child developing the disorder.

\section{Autosomal recessive disorders}

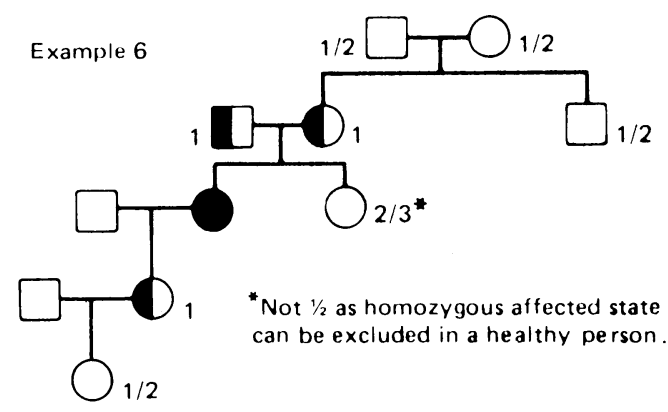

Recurrence of autosomal recessive disorders generally only occurs within a particular sibship. Many members of the family may be gene carriers, the risks of which are shown in example 6. 


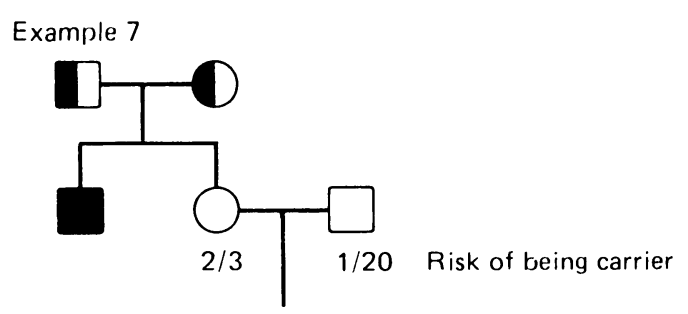

Risk of affected offspring

$2 / 3 \times 1 / 20 \times 1 / 4=1 / 120$
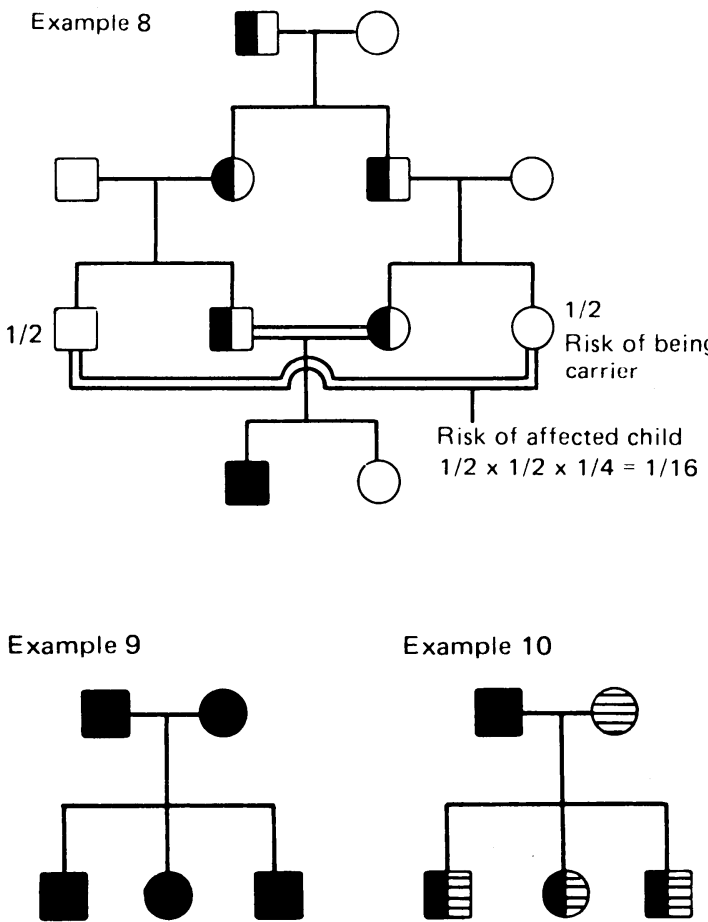

All offspring affected
Example 10

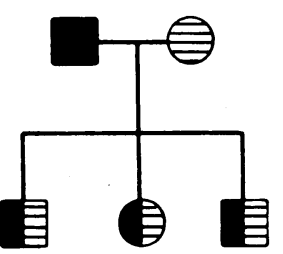

All offspring unaffected
The chance of a healthy sibling having affected children is low. The actual risk depends on the frequency of the gene in the general population. The risk for cystic fibrosis is shown in example 7. (In the general population about one in 20 people are gene carriers.)

When there is a tradition of consanguinity more than one marriage may be arranged between two families. If a consanguineous couple have a child affected by an autosomal recessive condition other family marriages may also be at risk of having affected offspring, as in example 8. The risk may not be high enough to prevent further planned marriages taking place, but if carrier state can be determined by specific tests this will help the families to make decisions and reassure the relatives who are not carriers.

When both parents are affected by a recessive condition the risks to the offspring will depend on whether the parents are homozygous for allelic or non-allelic genes as some autosomal recessive disorders, such as deafness, can be caused by different genes at separate loci. In example 9 both parents have the same form of recessive deafness and all their children will be affected. In example 10 the parents have different forms of recessive deafness due to genes at different loci. Their offspring will be heterozygous at both loci and therefore unaffected.

\section{$\mathrm{X}$ linked recessive disorders}

Example 11

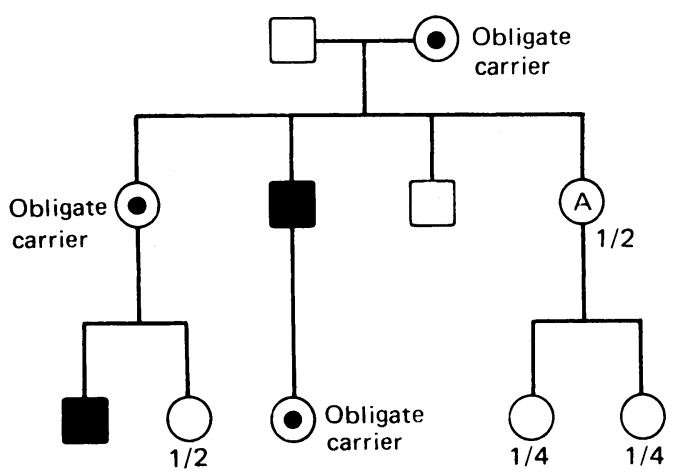

Example 12

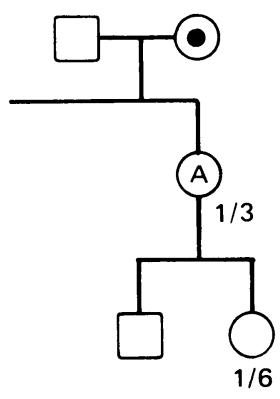

Example 13

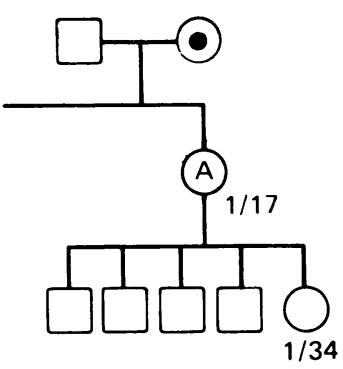

Calculation of risks in $\mathrm{X}$ linked recessive disorders is often complex, and the following examples illustrate some basic concepts without details of method. Referral to a specialist genetic centre is usually indicated for calculating carrier state, which depends on pedigree structure and results of specific tests. In families with $\mathrm{X}$ linked recessive disorders many female relatives are at risk of being carriers, as in example 11 .

If the female relative $A$ at risk in example 8 has any healthy sons this will reduce her risk to the values shown in examples 12 and 13 . 


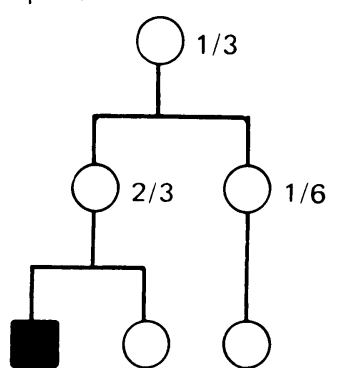

$1 / 3 \quad 1 / 12$

In lethal $\mathrm{X}$ linked disorders new mutations account for a third of all cases. A mother of an affected boy is therefore not always a carrier. Carrier risks in families in which there is an isolated case of such a disorder (for example, Duchenne muscular dystrophy) are shown in example 14. These risks would again be modified by the presence of any unaffected males in the pedigree as well as by the results of specific tests to detect carriers.

\section{Isolated cases}

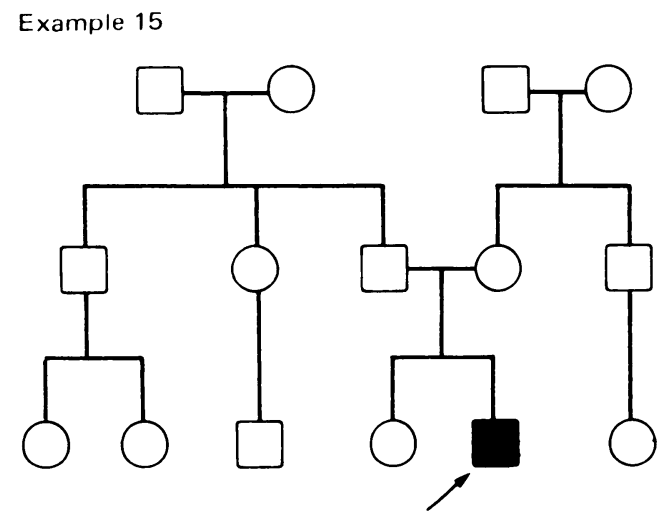

Pedigrees showing only one affected person are the type most commonly encountered in clinical practice (example 15). Various causes must be considered, and counselling in this situation depends entirely on reaching an accurate diagnosis in the affected person.

\section{Possible causes}

- Non-genetic

- Autosomal dominant: new mutation, non-paternity, or (rarely) parental germline mutation

- Autosomal recessive

- X linked recessive. May represent new mutation or inheritance from carrier mother

- Polygenic (multifactorial). Risk of recurrence generally low

- Chromosomal. Recurrence depends on type of abnormality, but generally low

Example 16

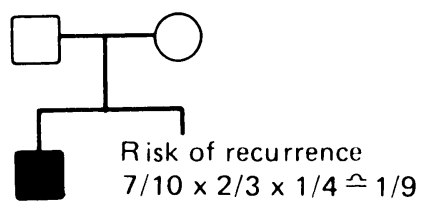

In example 16 calculation of the risk of recurrence after an isolated case of congenital deafness is based on the finding that when environmental causes are excluded $70 \%$ of cases are genetic, of which two thirds are recessive and a third are dominant.

Dr Helen M Kingston, MD, is consultant clinical geneticist at St Mary's Hospital, Manchester.

\section{ANY QUESTIONS}

Would the administration of potent oral steroids result in sufficient calciuria to
cause renal stones?

Glucocorticoids have complex effects on calcium and skeletal metabolism. Their administration in pharmacological amounts induces an acute increase in the urinary excretion of calcium. At the onset of their administration this may be partly related to a transient stimulation of intestinal calcium absorption, but calcium absorption is subsequently reduced and calciuria is principally due to the catabolic effects of corticosteroids on bone. Bone loss is rapid in the first few years of corticosteroid use, and thereafter the rate of loss falls as does the urinary excretion of calcium. The aetiology of calcium nephrolithiasis is also complex and multifactorial. The risk of renal stones increases progressively with increasing rates of excretion of calcium, ' and thus giving exogenous steroids presumably increases this risk. The older textbooks claim that renal stones occur in about a fifth of patients with longstanding Cushing's syndrome, ${ }^{2}$ but renal stones in some of these cases may have been due to multiple endocrine neoplasia (with hyperparathyroidism). Cushing's syndrome now rarely causes stones, ${ }^{2}$ though presumably the risk is increased, particularly in patients who may have other predisposing factors. - J A KANIS, reader in human metabolism and clinical biochemistry, Sheffield

1 Robertson WG. Urinary tract calculi. In: Nordin BEC, ed. Metabolic bone und stone disease. Edinburgh: Churchill Livingstone, 1984:271-326.

2 Williams RH. Textbook of endocrinology. 5th ed. Philadelphia: W B Saunders, 1974.
Has resistance been reported to multiple drug treatment for leprosy with rifampicin, clofazimine, and dapsone? If so, how is drug resistance measured?

There have been no published reports of resistance to the multiple drug treatment recommended since 1982 by the World Health Organisation (WHO) for leprosy, a treatment that is rapidly becoming universally accepted. Though drug resistance is unlikely after such a powerful antileprosy triple treatment, drug resistance in leprosy can be measured only clinically in view of the impracticability of routinely using mouse footpad infection as a direct measure of drug resistance of Mycobacterium leprae.

Drug resistance in leprosy is diagnosed as the cause of relapse or failure to improve after the standard WHO course of multiple drug treatment only when it can be confirmed beyond reasonable doubt that the drug dose and duration and regularity of treatment have been adhered to. The following criteria must be satisfied before drug resistance can be considered the cause of failure of multiple drug treatment in leprosy: (1) monthly attendance records to confirm attendance of the patient for supervised dose of rifampicin and topping up dose of clofazimine; (2) orange/red coloration of a urine sample taken between two and 10 hours after supervised dose of rifampicin; (3) reddish coloration of the skin within two to three months of the start of treatment confirms self administration of clofazimine; and (4) random urine tests for quantitative assessment of dapsone concentrations to confirm regular self administration of dapsone. $-\mathrm{R} \mathrm{J}$ W REES, research consultant, London

Ellard GA. Chemotherapy of leprosy. Br Med Bull 1983;44:775-90.

Ellard GA, Gammon PT, Helmy HS, Rees RJW. Urine tests to monitor the self-administration of dapsone by leprosy patients. Am F Trop Med Hyg 1974;23:464-70. 